\title{
Detecting Pancreatic Carcinoma in the Setting of Idiopathic Pancreatitis and Negative Cross-Sectional Imaging: Why EUS Is Useful
}

\author{
Jeffrey J. Easler ${ }^{1}$ \\ Published online: 23 October 2019 \\ (c) Springer Science+Business Media, LLC, part of Springer Nature 2019
}

The etiology of acute pancreatitis (AP) is uncertain in up to $30 \%$ of patients, even after a medical history, right upper quadrant ultrasound, and biochemical testing have been accomplished [1]. The term "idiopathic acute pancreatitis" (IAP) should be considered only after exclusion of alcohol abuse, biliary, or metabolic disease, and obvious medications as etiologies. Societal guidelines endorse advanced imaging such as EUS to evaluate patients with IAP. A recent meta-analysis demonstrated EUS to have a substantial diagnostic yield for IAP at $>60 \%$, with a statistically significant advantage over MRI/MRCP for detecting occult biliary disease (choledocholithiasis, microlithiasis, or sludge) [2].

A broad evaluation for additional toxic, metabolic, and/or morphologic contributors (TIGAR-O) is recommended for idiopathic chronic pancreatitis (ICP) patients. EUS in the setting of ICP is more often utilized to establish a diagnosis of $\mathrm{CP}$ and to discover pathology that drives therapeutic decision-making such as pancreatic duct stones and strictures [3].

While representing a minority of patients with IAP and ICP, the possibility of an unidentified pancreatic adenocarcinoma (PDAC) is a source of great concern and anxiety for patients and their physicians in this clinical setting. This concern is borne out of a relationship among AP, $\mathrm{CP}$, and PDAC now recognized and published in the literature. The strength of this relationship can be explored in several different ways.

Jeffrey J. Easler

jjeasler@iu.edu

1 Division of Gastroenterology and Hepatology, Indiana University School of Medicine, Indianapolis, USA

\section{In Patients with PDAC, How Frequently Is AP an Antecedent Event?}

AP is the initial event in the minority of PDAC patients $(5-11 \%)[4,5]$. A retrospective cohort study published by our center analyzed patterns of antecedent health care utilization in a cohort of PDAC patients $(n=1023)$ [5]. From data in the form of ICD-9 and 10 codes that was extracted from a healthcare database, $76 \%$ of patients had at least one healthcare encounter prior to their PDAC diagnosis. An incident diagnosis of AP was found in $11 \%$ during the preceding study term (36 months) and was associated with a pattern of consistent, higher utilization of inpatient and outpatient health care resources in the months and years preceding a PDAC diagnosis (OR 3.422, $p=<0.001)$. In this study, it appeared that some patients had a small, but clinically important window or lead time (months to years) between $\mathrm{AP}$ event(s) and PDAC diagnosis.

\section{What Is the Rate of Incident PDAC Diagnosis Following an Attack of AP or a Diagnosis of CP?}

A study utilizing data from the Swedish national health registry matched a cohort of patients with incident AP to patients without AP, reported a higher rate of PDAC following $\operatorname{AP}(1.1 \%$ vs. $0.1 \%)$. [6] The majority of PDAC in the AP cohort were diagnosed within the first 2 years $(89.3 \%)$ following the AP event. The risk for incident PDAC after AP declined to that observed in the non-AP cohort after 10 years. The risk of incident PDAC was also significantly greater in the matched AP age groups $>60$ years $(0.9-1 \%$ versus $0.4-0.6 \%$ ) and in patients with recurrent AP.

An elevated risk was also reported in this study for patients within the first 2 years of a new diagnosis of $\mathrm{CP}$ (HR 103.59, 95\% CI 69.25-154.98). This relationship has also been reported in other population studies. [7] This 
phenomenon may be related to PDAC masquerading as $\mathrm{CP}$ due to an overlap of symptoms such as abdominal pain or weight loss and shared radiographic features such as pancreatic atrophy or pancreatic duct dilation.

From these population studies, the following can be concluded: (1) a fraction of PDAC patients ( $\sim 5$ to $10 \%)$ have an antecedent history AP or CP; and (2) incident PDAC exists in a very small proportion of patients $(\sim 1 \%)$ with AP and CP. Nevertheless, incident PDAC does occur at a significantly higher rate in AP and in $\mathrm{CP}$ patients compared with the population at large; this risk is greatest within the first 2 years of an AP or CP diagnosis. Consequently, antecedent IAP may represent a window of opportunity for early diagnosis of PDAC.

\section{How is PDAC Detected in the Setting of AP and CP?}

With the ability to detect lesions with a diameter $<1 \mathrm{~cm}$, EUS is also reported to identify suspicious lesions in patients with clinical suspicion for neoplasia and negative antecedent cross-sectional imaging. [4, 8] In this month's issue of Digestive Diseases and Sciences, Bartell et al. examined the utility of EUS for the diagnosis of idiopathic pancreatitis. [9] The authors offer a retrospective, single center study that reports the rate of incident PDAC in patients referred for EUS in the setting of IAP and ICP. EUS referrals in all study patients $(n=565)$ followed cross-sectional imaging negative for a mass and occurred in the absence of obstructive jaundice. EUS was performed at an average interval of 104 days from presentation and identified incident pancreatic cancer in $5.3 \%$ of the cohort. The majority of lesions $(90 \% n=27 / 30)$ were PDAC with the incidence of malignancies evenly distributed across IAP and ICP indications for EUS in the cohort.

This study offers insight into the rate of incident PDAC in a population of patients: (1) with IAP or ICP in the absence of obstructive jaundice; (2) who have negative prior crosssectional imaging; and (3) who undergo EUS at an average (short) interval of 3 months from AP presentation. Based on Bartell's study, these circumstances appear to enrich the rate of incident PDAC in their population above that expected in the population at large and in IAP/ICP populations described in large database cohort studies. Nevertheless, the retrospective design of this study does limit the ability to understand the true denominator for an IAP/ICP cohort. Granular data regarding preceding cross-sectional studies (contrast versus non-contrast), the time from imaging studies to EUS, and the frequency of AP attacks preceding EUS would further clarify the sensitivity and perhaps the optimal timing of EUS in order to detect PDAC in an IAP/ICP cohort. The difference in timing of EUS between patients ultimately found to have PDAC versus those with a negative EUS study after IAP (104 versus 817.9 days, $p=0.003$ ) is also of interest. This suggests a selection bias for earlier EUS referral in the patients with IAP/ICP that were ultimately found to have PDAC, likely due to some combination of confounding variables (e.g., symptoms, family history) that further increased the care teams' suspicion for neoplasia and contributed to the interval enrichment for PDAC in this subgroup of the cohort.

An analysis for associations between pre-procedure demographics, imaging characteristics, and incident PDAC also revealed age $(p \leq 0.002)$ and pancreatic duct dilation on imaging $(p=0.009)$ to be significant factors. Yet, only a minority of patients with PDAC (26.7\%) displayed pancreatic duct dilation on imaging prior to EUS. It appears that the absence of pancreatic duct dilation is not a reliable negative predictor for neoplasia in this cohort. These findings are consistent with a retrospective study of 218 patients referred for EUS evaluation after AP following a negative evaluation for gallstone and alcohol etiology. PDAC was identified in $17 \%$ of these patients. Age ( $>50)$ and pre-procedure imaging findings of pancreatic atrophy and pancreatic duct dilation were associated with incident PDAC. Of note, the majority of patients diagnosed with PDAC had multiple risk factors identified by history (e.g., age, weight loss) or pre-EUS imaging (e.g., mass, atrophy) for neoplasia. [10] This suggests that no single pre-EUS characteristic alone is reliable in adjusting the pre-EUS suspicion for neoplasia up or down in an IAP/ICP cohort.

Bartell et al. also reported outcomes data in the PDAC arm of the cohort. More than 50\% of PDAC patients were diagnosed at Stage I/II, and of those with follow-up, more than 50\% ultimately underwent resection. This suggests EUS performed promptly after a diagnosis IAP/ICP may create an opportunity for detection of PDAC at an earlier, treatable stage and translate into a higher rate of curative resection.

Of interest is that $15 \%(n=4)$ of patients with PDAC were diagnosed after a second EUS study that followed a (false?) negative first examination. This occurred at a mean interval of 370 days from pancreatitis diagnosis; $75 \%$ (3/4) had Stage III/IV disease. Sensitivity of EUS in this cohort was therefore $85.2 \%$ for PDAC in the setting of IAP/ICP. It is conceivable that these lesions that were initially missed were T1 lesions, for which the sensitivity of EUS is reported to be $<75 \%$. [4] It would also be helpful to know what proportion of these patients missed at first pass EUS were referred for calcific ICP as the sensitivity of EUS for neoplasia in this setting is also diminished. Further research is required to understand the optimal approach for identifying very highrisk patients for interval PDAC after IAP/ICP and in whom to offer surveillance after negative advanced imaging.

In summary, Bartell et al. contribute to the literature supporting EUS for the evaluation of IAP/ICP, specifically as it 
pertains to detection of incident PDAC. While incidence of PDAC in an idiopathic pancreatitis population is low overall, it is greater than the population at large and in cohorts with pancreatitis by any known cause. Although pre-EUS suspicion for PDAC can be calibrated based on a combination of patient (e.g., age) and imaging characteristics (duct dilation), no single factor is reliable. The greatest argument for an EUS evaluation in order to identify PDAC in IAP/ ICP is that it may create an opportunity for diagnosis at an earlier stage and create an opportunity for resection with curative intent. For this opportunity and the reassurance that a negative EUS examination offers, many patients would be grateful for the referral.

\section{References}

1. Das R, Yadav D, Papachristou GI. Endoscopic treatment of recurrent acute pancreatitis and smoldering acute pancreatitis. Gastrointest Endosc Clin N Am. 2015;25:737-748.

2. Wan J, Ouyang Y, Yu C, et al. Comparison of EUS with MRCP in idiopathic acute pancreatitis: a systematic review and metaanalysis. Gastrointest Endosc. 2018;87(1180-1188):e9.

3. Conwell DL, Lee LS, Yadav D, et al. American pancreatic association practice guidelines in chronic pancreatitis: evidence-based report on diagnostic guidelines. Pancreas. 2014;43:1143-1162.
4. Singhi AD, Koay EJ, Chari ST, et al. Early detection of pancreatic cancer: opportunities and challenges. Gastroenterology. 2019;156:2024-2040.

5. Cote GA, Xu H, Easler JJ, et al. Informative patterns of healthcare utilization prior to the diagnosis of pancreatic ductal adenocarcinoma. Am J Epidemiol. 2017;186:944-951.

6. Sadr-Azodi O, Oskarsson V, Discacciati A, et al. Pancreatic cancer following acute pancreatitis: a population-based matched cohort study. Am J Gastroenterol. 2018;113:1711-1719.

7. Kirkegard J, Mortensen FV, Cronin-Fenton D. Chronic pancreatitis and pancreatic cancer risk: a systematic review and metaanalysis. Am J Gastroenterol. 2017;112:1366-1372.

8. Wang W, Shpaner A, Krishna SG, et al. Use of EUS-FNA in diagnosing pancreatic neoplasm without a definitive mass on CT. Gastrointest Endosc. 2013;78:73-80.

9. Bartell N, Bittner K, Vetter MS, et al. Role of endoscopic ultrasound in detecting pancreatic cancer missed on cross-sectional imaging in patients presenting with pancreatitis: a retrospective review. Dig Dis Sci. (Epub ahead of print). https://doi. org/10.1007/s10620-019-05807-z.

10. Tummala P, Tariq SH, Chibnall JT, et al. Clinical predictors of pancreatic carcinoma causing acute pancreatitis. Pancreas. 2013;42:108-113.

Publisher's Note Springer Nature remains neutral with regard to jurisdictional claims in published maps and institutional affiliations. 